\title{
Real-time monitoring of Pseudomonas aeruginosa biofilm formation on endotracheal tubes in vitro
}

\author{
Eva Pericolini ${ }^{1 *+} \mathbb{D}$, Bruna Colombari ${ }^{1 \dagger}$, Gianmarco Ferretti ${ }^{1}$, Ramona Iseppi ${ }^{2}$, Andrea Ardizzoni ${ }^{1}$, Massimo Girardis ${ }^{1}$, \\ Arianna Sala', Samuele Peppoloni ${ }^{1}$ and Elisabetta Blasi ${ }^{1}$
}

\begin{abstract}
Background: Pseudomonas aeruginosa is an opportunistic bacterial pathogen responsible for both acute and chronic infections in humans. In particular, its ability to form biofilm, on biotic and abiotic surfaces, makes it particularly resistant to host's immune defenses and current antibiotic therapies as well. Innovative antimicrobial materials, like hydrogel, silver salts or nanoparticles have been used to cover new generation catheters with promising results. Nevertheless, biofilm remains a major health problem. For instance, biofilm produced onto endotracheal tubes (ETT) of ventilated patients plays a relevant role in the onset of ventilation-associated pneumonia. Most of our knowledge on Pseudomonas aeruginosa biofilm derives from in vitro studies carried out on abiotic surfaces, such as polystyrene microplates or plastic materials used for ETT manufacturing. However, these approaches often provide underestimated results since other parameters, in addition to bacterial features (i.e. shape and material composition of ETT) might strongly influence biofilm formation.
\end{abstract}

Results: We used an already established biofilm development assay on medically-relevant foreign devices (CVC catheters) by a stably transformed bioluminescent (BLI)-Pseudomonas aeruginosa strain, in order to follow up biofilm formation on ETT by bioluminescence detection. Our results demonstrated that it is possible: i) to monitor BLI-Pseudomonas aeruginosa biofilm development on ETT pieces in real-time, ii) to evaluate the three-dimensional structure of biofilm directly on ETT, iii) to assess metabolic behavior and the production of microbial virulence traits of bacteria embedded on ETT-biofilm.

Conclusions: Overall, we were able to standardize a rapid and easy-to-perform in vitro model for real-time monitoring Pseudomonas aeruginosa biofilm formation directly onto ETT pieces, taking into account not only microbial factors, but also ETT shape and material. Our study provides a rapid method for future screening and validation of novel antimicrobial drugs as well as for the evaluation of novel biomaterials employed in the production of new classes of ETT.

Keywords: Pseudomonas aeruginosa, Biofilm, Real-time monitoring, Endotracheal tube, Bioluminescence

\section{Background}

It is known that microbial biofilm frequently forms on the surface of endotracheal tubes (ETT) implanted in patients receiving assisted ventilation [1-4]. In particular, biofilm occurs onto more than $90 \%$ of ETT within 7 days from insertion, since standard cleaning practices are not able to fully clear bacterial slime $[5,6]$. As a consequence,

\footnotetext{
*Correspondence: eva.pericolini@unimore.it

${ }^{\dagger}$ Eva Pericolini and Bruna Colombari contributed equally to this work.

'Department of Surgical, Medical, Dental and Morphological Sciences with interest in Transplant, Oncological and Regenerative Medicine, University of Modena and Reggio Emilia, Modena, Italy
}

Full list of author information is available at the end of the article
ETT-associated biofilm increases the risk of upper respiratory tract infections, as well as device occlusion and wound infections, among other complications $[7,8]$. Biofilm on ETT is recognized to be one of the main causes of ventilator-associated pneumonia (VAP) $[9,10]$. The latter is commonly caused by the opportunistic pathogen Pseudomonas aeruginosa ( $P$. aeruginosa) [11]. $P$. aeruginosa is a Gram negative bacterium that possesses several virulence tracts, some of which are cell-associated moieties (like flagella, pili, lectins, alginate/biofilm, lipopolysaccharide) while some others are secreted (namely proteases, hemolysins, cytotoxin, pyocyanin, siderophores, exotoxin A, exoenzyme

(c) The Author(s). 2018 Open Access This article is distributed under the terms of the Creative Commons Attribution 4.0 International License (http://creativecommons.org/licenses/by/4.0/), which permits unrestricted use, distribution, and 
$\mathrm{S}$, exoenzyme $\mathrm{U}$, etc.) [12]. Furthermore, $P$. aeruginosa is one of the greatest biofilm producers.

Soon after intubation, bacteria can adhere and multiply on the ETT surface to form biofilm. From there, entry of opportunistic pathogenic bacteria is facilitated so that the lower respiratory tract as well as the lung parenchyma are often involved [2, 11]. Notoriously, biofilm represents a complex and tightly adherent microbial community, embedded in an abundant matrix of hydrated extracellular polymeric substance (EPS), primarily composed of polysaccharides, proteins, nucleic acids and lipids [13]. From biofilm, microbial cells detach and easily get access to the lower airways through ventilator gas flow and aspiration [2]. Therefore, biofilm on ETT provides a persistent reservoir of pathogens likely responsible for VAP $[3,10,11]$. $P$. aeruginosa biofilm structure and stability are determined by at least three different polysaccharides, namely alginate, Pel and Psl $[14,15]$. In particular, alginate is able to stabilize biofilm structure and to contribute to water retention and nutrients accumulation inside the matrix [16]. Another relevant component of $P$. aeruginosa biofilm is extracellular DNA (eDNA), which is known to play a key role in the onset and growth of $P$. aeruginosa biofilm, thanks to its ability to act as a cell to cell interconnection compound $[13,17,18]$ and to be precious nutrient source for the embedded bacteria [19].

Once established, biofilm can resist to antibiotics and host immune response [20-23]. New chemical and mechanical approaches are currently under study to fight biofilm formation on ETT. The use of modified ETT (e.g. cuffed ETT and silver or other nanoparticle-coated ETT) have been shown to decrease the incidence of VAP in adults [24, 25]. Nevertheless, biofilm remains a serious health problem, requiring the development of efficacious preventive and therapeutic anti-biofilm approaches. In addition to bacterial factors, a largely underestimated feature, i.e. the combination of ETT shape and material composition, may strongly influence biofilm formation [26]. Indeed, most of our present knowledge on $P$. aeruginosa biofilm development onto ETT originates from in vitro studies on abiotic substrates, such as polystyrene wells or plastics per se, without taking into account other structural and three-dimensional parameters.

In the present work, by using a bioluminescent strain of $P$. aeruginosa, we describe a rapid and easy-to-perform in vitro system for real-time monitoring $P$. aeruginosa biofilm formation, directly onto ETT. Through this procedure, it was also possible to evaluate the biofilm three-dimensional structure, the metabolic behaviour and the expression of microbial virulence traits of bacteria embedded within ETT-biofilm.

Therefore, our innovative approach allows us to study biofilm formation directly onto medical devices, taking into account not only microbial factors but also the combination of ETT shape and material. In our hands, we believe that such model mimics much more closely the process of biofilm formation occurring in the clinical setting.

\section{Methods \\ Pseudomonas aeruginosa strains}

The following strains were used: the bioluminescent $P$. aeruginosa (P1242) (BLI-Pseudomonas) and $P$. aeruginosa PAO1 (ATCC 15692) (wild type (WT)-Pseudomonas). As detailed elsewhere, BLI-Pseudomonas expressed the luciferase gene and luciferase substrate under the control of a constitutive P1 integron promoter [27].

\section{Pseudomonas aeruginosa culture conditions and cell growth}

Bacteria from $-80{ }^{\circ} \mathrm{C}$ glycerol stocks were initially seeded onto Tryptic Soy Agar (TSA) plates and incubated overnight at $37^{\circ} \mathrm{C}$; then, a fresh single colony was collected, inoculated into $10 \mathrm{ml}$ of Tryptic Soy Broth (TSB) and cultured overnight at $37^{\circ} \mathrm{C}$. The culture was then washed and inoculated into fresh medium (TSB plus $2 \%$ sucrose) at a dilution 1:10. To create a bacterial growth curve, the optical density at $595 \mathrm{~nm}\left(\mathrm{OD}_{595}\right)$ was determined every $20 \mathrm{~min}$ using a spectrophotometer (Tecan Sunrise $^{\mathrm{Tm}}$ ). After each reading, $100 \mu \mathrm{l}$ were collected and plated onto TSA in order to determine the number of Colony Forming Units (CFU). For all the experiments, starting bacterial suspension was adjusted at $5 \times 10^{4} \mathrm{CFU} / \mathrm{ml}$ in TSB with $2 \%$ sucrose. To quantify the bioluminescence emission by BLI-Pseudomonas in the experimental groups, a calibration curve was generated in the microtiter plate. In particular, serial dilutions (starting from $1 \times 10^{7} / \mathrm{ml}$ ) of bacterial suspension in TSB with $2 \%$ sucrose were prepared and $100 \mu \mathrm{l}$ of each dilution was seeded in a black transparent-well microtiter plate. The plate was immediately read by using Victor $^{\mathrm{rm}}$ X Light 2030 Luminescence reader (Perkin Elmer).

\section{Endotracheal tube (ETT) pieces preparation}

Twenty-four hours before each experiment, a sterile disposable paediatric endotracheal tube (ETT) (RUSCH $3.0 \mathrm{~mm}-5.0 \mathrm{~mm}$ ) was cut under a biological safety cabinet (to maintain sterile conditions), in pieces of $0.5 \mathrm{~cm}$ length, as previously described by Kucharikova S. et al., with minor modifications [28] (Additional file 1). A maximum of 4 ETT pieces were placed into $1.5 \mathrm{ml}$ microcentrifuge tubes, covered with foetal bovine serum (FBS) and vortexed. Then, further 100-200 $\mu$ l of FBS were added to each tube to completely cover all ETT pieces, before being incubated at $37{ }^{\circ} \mathrm{C}$ overnight in static conditions. 


\section{Biofilm formation on ETT pieces}

In order to allow biofilm formation on ETT pieces, $200 \mu \mathrm{l}$ of overnight cultures of BLI-Pseudomonas or WT-Pseudomonas (both at $5 \times 10^{4} / \mathrm{ml}$ ) in TSB with $2 \%$ sucrose were seeded in 96 well-plates, containing 1 ETT piece/well, either in the presence or absence of gentamicin $(4 \mu \mathrm{g} / \mathrm{ml})$; the plates were then incubated at $37{ }^{\circ} \mathrm{C}$ for $90 \mathrm{~min}$ (adhesion period). After incubation, the ETT pieces were washed twice with PBS at room temperature (RT), transferred to new wells and incubated for 12, 24 or $48 \mathrm{~h}$ in fresh TSB with $2 \%$ sucrose with or without gentamicin $(4 \mu \mathrm{g} / \mathrm{ml})$ at $37{ }^{\circ} \mathrm{C}$ plus $5 \% \mathrm{CO}_{2}$. Unless otherwise specified, every $24 \mathrm{~h}$ the culture medium was replaced with fresh medium with or without gentamicin. After 12, 24 or $48 \mathrm{~h}$ of incubation, ETT pieces were washed twice with PBS at RT and biofilms were analyzed both by our innovative method and by a standard methods (see below).

\section{Real-time monitoring of biofilm formation on ETT pieces} by bioluminescence and CFU analysis

After biofilm formation on ETT pieces, as described above, the bioluminescence was measured by using the Victor $^{\text {rn }}$ X Light 2030 Luminescence reader (Perkin Elmer). Data is shown as Relative Luminescence Units (RLU).

In selected experiments, the ETT pieces, treated as above described, were washed twice with PBS at RT, transferred to a microcentrifuge tubes containing PBS $(1 \mathrm{ml})$, sonicated for $15 \mathrm{~min}$ at $30,000 \mathrm{~Hz}$ in a water bath sonicator, then placed on ice, vortexed for $20 \mathrm{~s}$ and placed again on ice. Subsequently, $100 \mu \mathrm{l}$ of each microbial suspension, appropriately diluted, were plated onto TSA and incubated for $48 \mathrm{~h}$ at $37{ }^{\circ} \mathrm{C}$. After incubation, the CFU were counted and data was expressed as $\mathrm{CFU} / \mathrm{ml}$.

\section{Evaluation of biofilm formation on ETT pieces by transmitted-illumination confocal microscopy and crystal violet staining}

After the 90 min of adhesion period (T0) and 24 and $48 \mathrm{~h}$ of exposure to BLI-Pseudomonas as above described, the ETT pieces were washed twice with PBS at RT and fixed with paraformaldehyde (PFA) for $30 \mathrm{~min}$ at $4{ }^{\circ} \mathrm{C}$, washed twice with PBS and then analyzed by transmitted-illumination confocal microscopy Nikon LV 150 Confovis Microscope. In selected experiments, after the 12,24 and $48 \mathrm{~h}$ of exposure to BLI-Pseudomonas as described above, the ETT pieces were washed twice with PBS at RT, transferred to new wells and then stained with $2 \%$ crystal violet $(\mathrm{CV})$ to quantify total biofilm mass directly on the ETT. This was done according with Stepanović's protocol, with minor modifications [29]. Prior to OD evaluation, the ETT pieces were removed from the wells and the OD were measured on the liquid phase only. The OD values were measured at $570 \mathrm{~nm}$ by using Sunrise ${ }^{\text {Tx }}$ spectrophotometer (Tecan).
Analysis of alive/dead cells embedded in ETT biofilm

To discriminate alive/dead microbial cells directly within biofilm formed on ETT pieces, after $48 \mathrm{~h}$ of exposure to BLI-Pseudomonas in the presence or absence of gentamicin $(4 \mu \mathrm{g} / \mathrm{ml})$, as described above, the ETT pieces were washed twice with PBS at RT, transferred to new wells containing $200 \mu \mathrm{l}$ of PBS and stained by using the "live/ dead imaging kit" (Thermo Fisher Scientific) that employs propidium iodide (PI) to label dead cells and 5(6)-carboxyfluorescein diacetate (CFDA) to label alive cells. The staining protocol was conducted according to the Manufacturer's instruction. After 15 min of incubation at $37^{\circ} \mathrm{C}$, the ETT pieces were washed twice with PBS and the fluorescence emission (PI excitation/emission: 528/ 645; CFDA excitation/emission: 485/528) was analysed by a multi-well fluorescence plate reader (Synergi HTX, BIOTEK). The results were expressed as relative fluorescence units (RFU). In parallel, microbial bacterial cultures (used as a control) were grown in planktonic form for $48 \mathrm{~h}$ with or without gentamicin, washed, centrifuged, resuspended in fresh medium and stained with the same kit.

\section{Quantification of eDNA and pyoverdine in cell-free supernatants from ETT biofilm}

For the analysis of eDNA and pyoverdine in cell-free supernatants, after the adhesion period, ETT pieces were incubated for 24 or $48 \mathrm{~h}$ in TSB with $2 \%$ sucrose with or without gentamicin $(4 \mu \mathrm{g} / \mathrm{ml})$. Medium was never replaced during the incubation period, which was carried out at $37{ }^{\circ} \mathrm{C}$ plus $5 \% \mathrm{CO}_{2}$. Then, cell-free culture supernatants were collected and tested for eDNA and pyoverdine content. In detail, the ETT pieces were removed and the supernatants were collected and centrifuged twice at 10,000 rpm for $15 \mathrm{~min}$ in order to remove the remaining bacteria. To exclude residual viable bacteria, $50 \mu \mathrm{l}$ of the supernatants were seeded onto TSA plates and incubated for $48 \mathrm{~h}$ at $37^{\circ} \mathrm{C}$ under aerobic conditions; no bacterial CFU on TSA plates were ever observed. To quantify eDNA concentration in the cell-free supernatants, $100 \mu \mathrm{l}$ of supernatants were incubated with PI $(1 \mu \mathrm{g} /$ $\mathrm{ml}$ ) for $15 \mathrm{~min}$ at $37^{\circ} \mathrm{C}$; then, fluorescence emission was quantified with a multi-well fluorescence plate reader (Synergi HTX, BIOTEK) (excitation/emission: 528/645).

In parallel, pyoverdine release was quantified in $100 \mu \mathrm{l}$ of the same culture supernatants (excitation/emission: $360 / 460)$, according to a standard protocol [30]. The measured amounts of eDNA and pyoverdine were plotted as RFU mean of triplicate samples \pm SEM.

Moreover, microbial bacterial cultures (used as a control) were grown in planktonic form for 24 and $48 \mathrm{~h}$ with or without gentamicin $(4 \mu \mathrm{g} / \mathrm{ml})$ and cell-free supernatants were analysed for the presence of both eDNA and pyoverdine, as above described. 


\section{Quantification of biofilm on coverslips}

To produce biofilm on coverslips, $1 \mathrm{ml}$ of overnight cultures of BLI-Pseudomonas $\left(5 \times 10^{4} / \mathrm{ml}\right)$ in TSB with $2 \%$ sucrose were seeded in 6-well-chamber slides containing coverslips in the presence or absence of gentamicin $(4 \mu \mathrm{g} / \mathrm{ml})$ and incubated for 24,48 or $72 \mathrm{~h}$ at $37{ }^{\circ} \mathrm{C}$ plus $5 \% \mathrm{CO}_{2}$. Every $24 \mathrm{~h}$, the medium was replaced with fresh medium with or without gentamicin $(4 \mu \mathrm{g} / \mathrm{ml})$. After 24,48 or $72 \mathrm{~h}$ of incubation, coverslips were washed twice with PBS at RT, air-dried, transferred to a microscope slide and analyzed, as previously described [31], with a Nikon Eclipse 90i imaging system equipped with Nomarski DIC optics (Nikon Instruments Inc. USA) (40X). Images were acquired by a DS-2Mv Nikon digital camera and analysed using the Nikon NISELEMENTS version D3.1 software. The thickness of the biofilm was measured and expressed in $\mu \mathrm{m}$.

\section{Biofilm-related genes}

After $24 \mathrm{~h}$ of exposure to BLI-Pseudomonas in the presence or absence of gentamicin $(4 \mu \mathrm{g} / \mathrm{ml})$, as described above, the ETT pieces were washed twice with PBS at RT, transferred in a microcentrifuge tubes containing PBS $(1 \mathrm{ml})$, sonicated for $15 \mathrm{~min}$ at $30,000 \mathrm{~Hz}$ in a water bath sonicator, then placed on ice, pipetting up and down ten times. Then, $100 \mu \mathrm{l}$ from each tube as well as of $100 \mu \mathrm{l}$ of an overnight fresh culture of BLI-Pseudomonas (both $1 \times 10^{6}$ cells) were used for DNA extraction by the rapid alkaline lysis method, as previously described [32]. The lasR-lasI quorum sensing (QS) genes [33], the las $B$, tox $A$ and the $\operatorname{alg} D$ genes [34], as well as proC housekeeping gene [35], were determined by polymerase chain reaction (PCR) performed and analysed as described previously [36], using primers reported elsewhere [33-35].

\section{Statistical analyses}

Statistical analysis was conducted using GraphPad Prism 7.0 software. Data depicted in Figures are the mean \pm standard error (SEM) from replicate samples of 3-7 different experiments. Statistical analysis was carried out with either two-tailed Student's t-test or one-way ANOVA with Bonferroni's post-hoc test. Values of $p<0.05$ were considered significant.

\section{Results}

Real-time monitoring of BLI-Pseudomonas biofilm on ETT To investigate the possibility of real-time monitoring biofilm formation directly onto ETT pieces, we followed the bioluminescence emission from each single ETT piece exposed to BLI-Pseudomonas during a 12, 24 and $48 \mathrm{~h}$ incubation period. In parallel, the WT-Pseudomonas was used as negative control. Box plots in Fig. 1a show that the bioluminescence signal, expressed as RLU, significantly increased over the time. The treatment with gentamicin strongly affected biofilm formation on ETT pieces, at each time point tested. As expected, no bioluminescence signal was ever observed when using WT-Pseudomonas (data not shown). Figure $1 \mathrm{~b}$ shows the results of representative experiments where groups of twelve samples (treated or not with gentamicin) were analysed in kinetics for bioluminescence emission. Each piece was marked with a different colour, so that it was possible to follow biofilm formation in each individual ETT piece over time. In selected experiments, we quantified the CFU (by standard method) from biofilm grown on ETT pieces, using the same experimental conditions. The results, depicted in Fig. 1c, showed that the number of CFU had a trend similar to that observed by bioluminescence analysis; nevertheless, the RLU results seemed to be more accurate in discriminating kinetic differences between the experimental groups. Figure 1d shows the kinetics of biofilm formation, assessed by means of CFU, on ETT pieces treated and untreated with gentamicin.

Using both tests (i.e. bioluminescence and CFU), no significant differences were observed between 24 and $48 \mathrm{~h}$, suggesting that, in our experimental conditions, the maximum of biofilm biomass was achieved after $24 \mathrm{~h}$. Gentamicin treatment significantly affected biofilm formation, both in terms of RLU and CFU (Fig. 1). Preliminary experiments, performed to construct a calibration curve, indicated that the analytical sensitivity of the bioluminescence signal from the BLI-Pseudomonas cells was more than 1000 bacterial cells (Additional file 2).

\section{Topographic analysis and evaluation of biofilm biomass on ETT}

To further characterize the biofilm structure on ETT, we performed a topographic analysis (3D mapping) of a 0 , 24 and 48 h-old biofilm produced on ETT pieces, by transmitted-illumination confocal microscopy. The results show the absence of biofilm at $0 \mathrm{~h}$ (Fig. $2 \mathrm{a}$ and $\mathrm{b}$ ). Moreover, Fig. $2 \mathrm{c}$ and e show the tridimensional architecture of the biofilm after 24 and $48 \mathrm{~h}$ of incubation with BLI-Pseudomonas, respectively. In addition, to better evaluate biofilm structure and thickness, a topographical reconstruction of the surface by means of a false color scale was carried out (Fig. 2b, $d$ and f). Finally, a 3D reconstruction of $24 \mathrm{~h}$ and $48 \mathrm{~h}$-old biofilm could be obtained by means of a mathematical linearization of the ETT surfaces, as shown in Fig. 2, C-inset and E-inset.

The biofilm on coverslips was already detectable at $24 \mathrm{~h}$ and it kept on growing significantly up to $72 \mathrm{~h}$ of culture, when it reached $65 \mu \mathrm{m}$ of thickness. No biofilm was produced on gentamicin-treated coverslip samples (Additional file 3). Crystal violet staining of 12, 24 and 48 h-old biofilm, directly on ETT pieces, showed a significant increase of biofilm mass over time. As expected, 

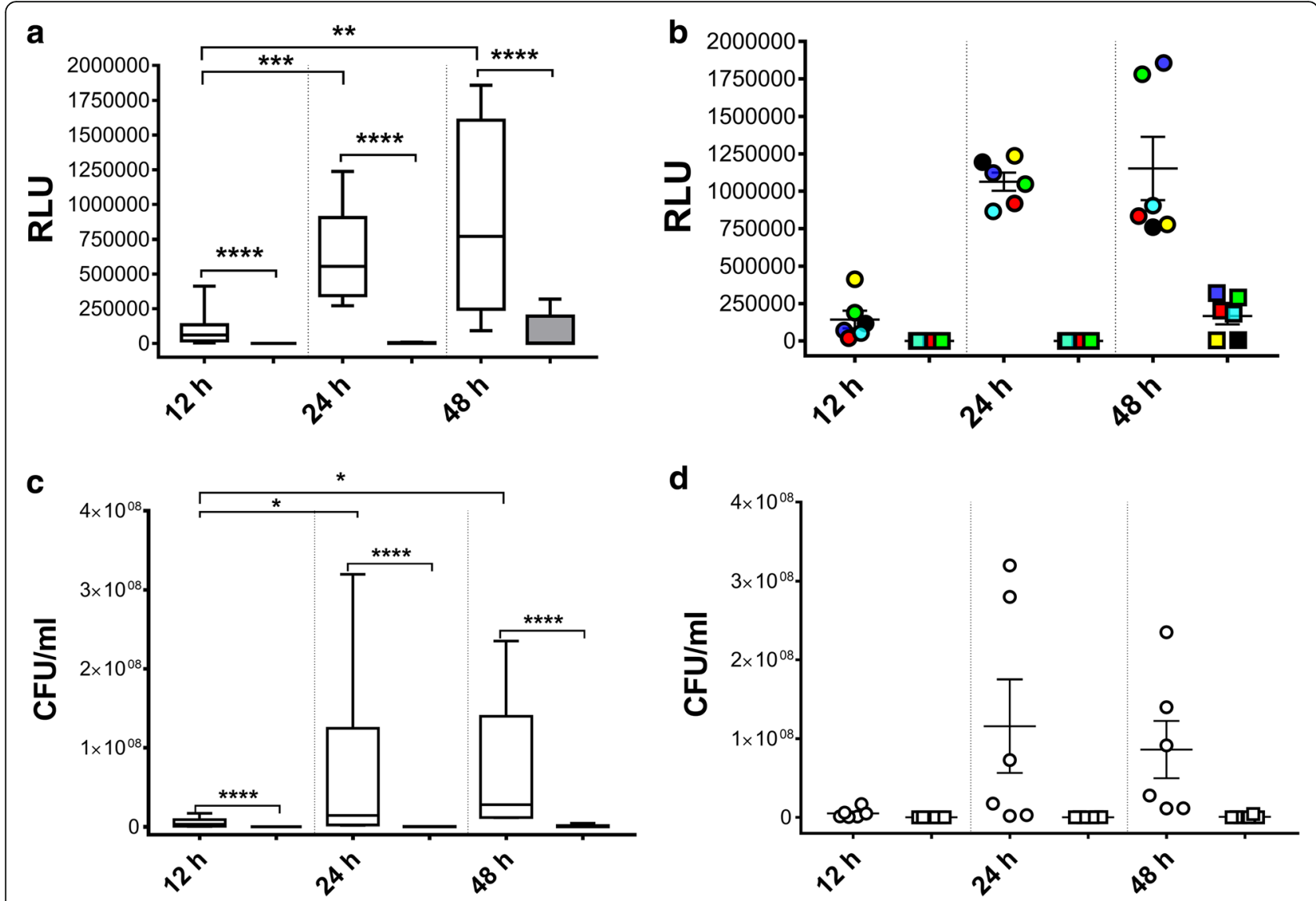

Fig. 1 Real-time evaluation of biofilm formation on ETT pieces and CFU counts. Box plots of RLU (a) and of CFU/ml (c) of BLI-Pseudomonas biofilm on ETT pieces, untreated (white box plots) or treated (grey box plots) with gentamicin after 12, 24 or $48 \mathrm{~h}$ of incubation. Mean $+/-$ SEM of RLU (b) or CFU/ml (d) analysis from 12 different ETT pieces. Circles or squares indicate the ETT pieces untreated or treated with gentamicin, respectively. Each ETT piece in panel $\mathbf{b}$ has a different colour: this allows us to follow the development of biofilm over time. Values of $p<0.05(*)$, $p<0.01\left(^{(*)}\right), p<0.001\left(^{* * *}\right)$ and $p<0.0001\left(^{(* * *)}\right.$ were considered significant

treatment with gentamicin significantly affected biofilm formation, being this effect particularly evident after 24 and $48 \mathrm{~h}$ (Fig. 3).

\section{Evaluation of both living and dead cells embedded in ETT biofilm}

In order to demonstrate the versatility of our model, we investigated the metabolic changes in biofilm following gentamicin treatment, as a prototype of response to different therapies directly onto ETT. In particular, ETT pieces were incubated for $48 \mathrm{~h}$ with BLI-Pseudomonas either in the presence or in absence of gentamicin, and then stained with PI and CFDA to discriminate live from dead cells. As shown in Fig. 4, the addition of gentamicin strongly reduced the number of metabolically active and viable bacteria embedded in the polymer matrix of biofilm, as assessed by RFU (panel a) and percentage of alive/dead cells ratio (panel b), respectively. According to the evidence that the eDNA released from dead cells remains embedded within biofilm matrix [17], the signal of gentamicin-treated biofilm dead cells is much higher than the signal of gentamicin-treated planktonic dead cells.

Evaluation of $P$. aeruginosa virulence traits in ETT biofilm It has been widely demonstrated that eDNA is a relevant component of $P$. aeruginosa biofilm, essential for the onset and stability of this sessile community $[17,18]$. Hence, we analyzed eDNA directly on cell-free supernatants of ETT exposed to BLI-Pseudomonas. As shown in Fig. 5a, a consistent production of eDNA was observed 24 and $48 \mathrm{~h}$ later; moreover, as expected, eDNA production was significantly impaired following the gentamicin treatment. A minimal release of eDNA could be detected only from 24 h-old planktonic cultures.

In our model, we also analyzed pyoverdine release. Indeed, pyoverdine is a key pigment for in vivo iron gathering and virulence expression by $P$. aeruginosa [37], and thus it contributes to promote the formation of biofilm [38]. Our results showed that significant levels 

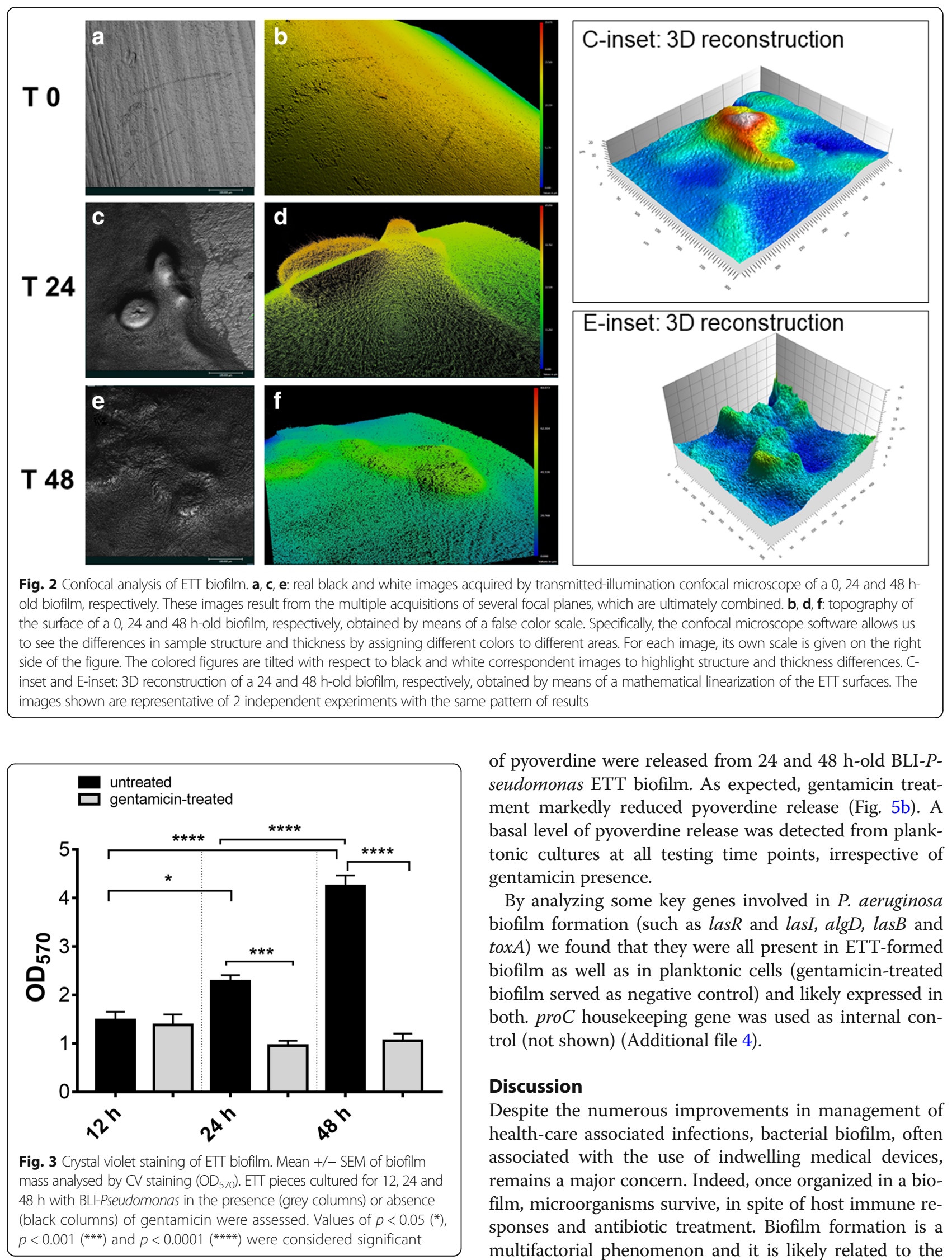

of pyoverdine were released from 24 and $48 \mathrm{~h}$-old BLI-Pseudomonas ETT biofilm. As expected, gentamicin treatment markedly reduced pyoverdine release (Fig. 5b). A basal level of pyoverdine release was detected from planktonic cultures at all testing time points, irrespective of gentamicin presence.

By analyzing some key genes involved in $P$. aeruginosa biofilm formation (such as lasR and lasI, $\operatorname{alg} D$, lasB and toxA) we found that they were all present in ETT-formed biofilm as well as in planktonic cells (gentamicin-treated biofilm served as negative control) and likely expressed in both. proC housekeeping gene was used as internal control (not shown) (Additional file 4).

\section{Discussion}

Despite the numerous improvements in management of health-care associated infections, bacterial biofilm, often associated with the use of indwelling medical devices, remains a major concern. Indeed, once organized in a biofilm, microorganisms survive, in spite of host immune responses and antibiotic treatment. Biofilm formation is a multifactorial phenomenon and it is likely related to the 


\section{alive cells $\square$ dead cells}

a

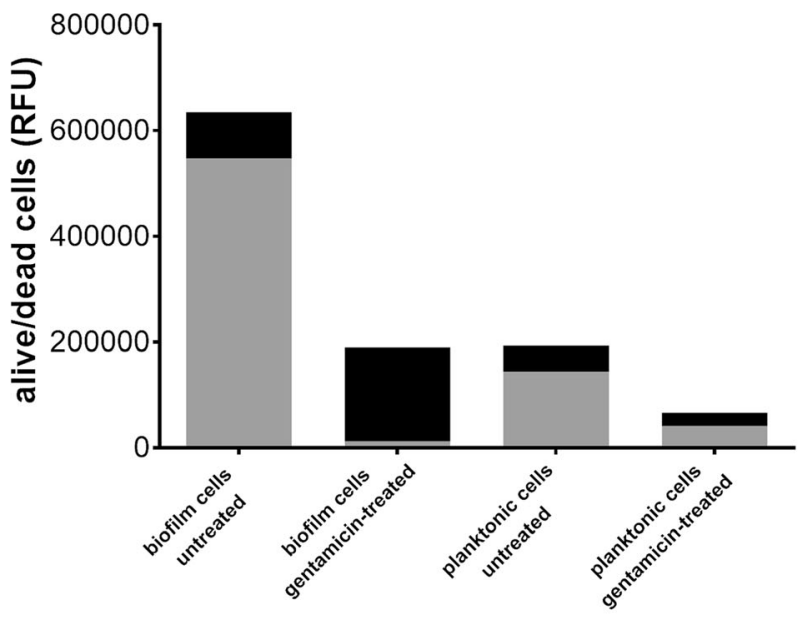

b

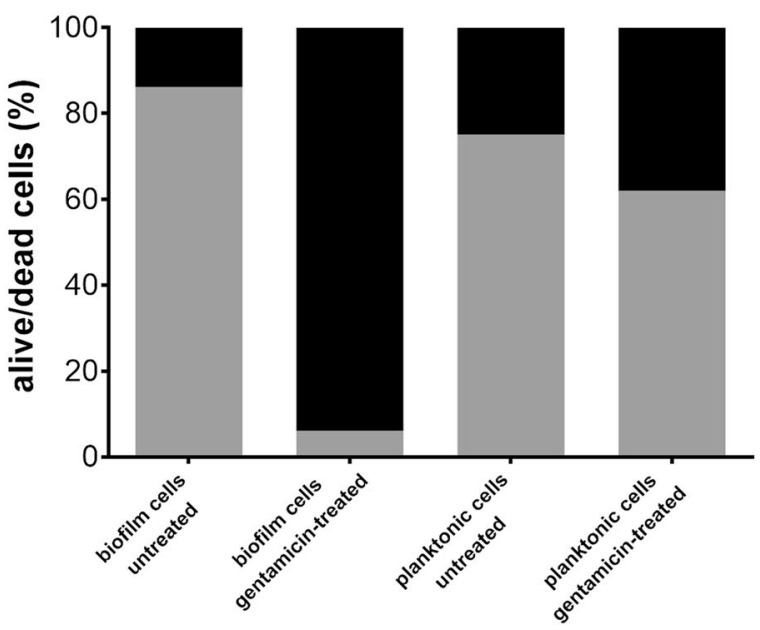

Fig. 4 Quantification of alive/dead cells embedded in ETT biofilm. Relative Fluorescence Units (RFU) mean (a) and percentage (b) of alive (grey) and dead (black) cells into ETT 48 h-old BLI-Pseudomonas biofilm and planktonic cultures, treated or not with gentamicin. The data shown are representative of three independent experiments, which provided similar pattern of results

production of a resistant EPS coating, to the reduction of microbial metabolism within the biofilm, and to the capacity of bacteria to share important drug-resistance genes [39]. Among numerous devices, ETT are especially susceptible to biofilm formation. They easily undergo microbial colonization and biofilm formation on their own surface, because of the concomitant skin/mucosa barrier breakdown as well as ETT exposure to respiratory mucus and blood that may further facilitate bacterial binding to such abiotic surface [7, 40, 41]. Finally, biofilm formation on ETT is widely feared since it provides a hidden bacterial reservoir that may delocalize and cause VAP among mechanically ventilated patients [10].
Most of our current knowledge of $P$. aeruginosa biofilm formation on medical devices, such as ETT, derives from in vitro studies on polystyrene or rough plastic sections [30, 42, 43]. Such models suffer from a relevant limitation, i.e. an underestimation of the role played by both device shape and material of ETT combined. These features, in addition to bacterial factors, may affect biofilm formation in vivo. For this reason, here, we describe a rapid and easy-to-perform in vitro method, which allows for a real-time evaluation of all the phases of $P$. aeruginosa biofilm formation onto a medical device. This method is designed to mimic, as close as possible, the real-life conditions, where the combination of microbial virulence
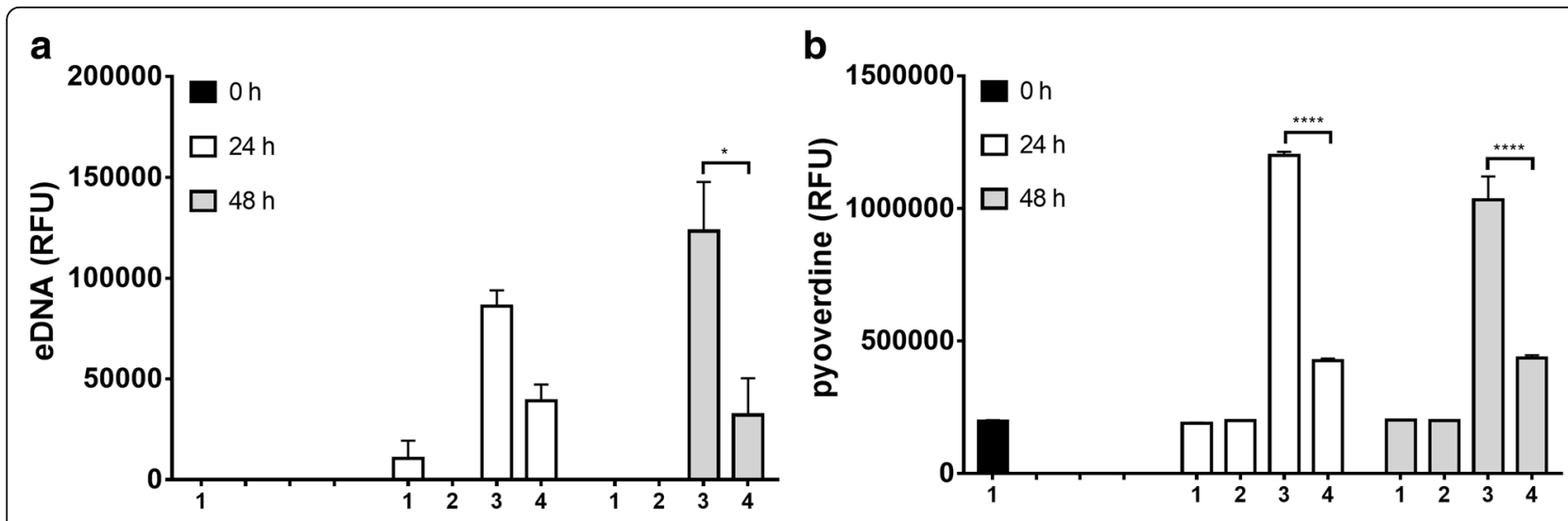

Fig. $\mathbf{5}$ eDNA and pyoverdine determination in cell-free supernatants from ETT biofilmRFU mean +/- SEM of eDNA (a) and pyoverdine (b) production in cell-free supernatants of BLI-Pseudomonas biofilm on ETT pieces and planktonic cultures, untreated or treated with gentamicin, after 0, 24 or 48 h of incubation. 1: untreated planktonic cells 2: gentamicin-treated planktonic cells 3: untreated biofilm 4: gentamicin-treated biofilm ${ }^{* * *} p<0.001$ and ${ }^{*} p<0.05$; gentamicin-treated samples vs untreated samples 
factors and device shape and material all play a crucial role in biofilm formation.

On a previous work, Kucharikova and colleagues [28], have monitored real-time biofilm formation onto central venous catheter $(\mathrm{CVC})$ pieces, using a well-established engineered strain of bioluminescent C. albicans (gLUC59) [44]. Starting from these data, here we have used an engineered strain of bioluminescent $P$. aeruginosa (BLI-Pseudomonas) to follow up in real-time biofilm formation on ETT pieces. Although the two models are conceptually quite similar, the BLI-Pseudomonas employed in this study offers a substantial advantage: unlike bioluminescent $C$. albicans, which needs the exogenous administration of the specific substrate (coelenterazine) to emit a quantifiable luminescence [44], BLI-Pseudomonas can be used and assessed as it is. BLI-Pseudomonas bears and constitutively expresses both the gene and the substrate necessary for the bioluminescence emission, as a results of the introduction of a specific $l u x$ operon $[27,45]$. This peculiarity renders the system rapid and ready-to-use. The possibility to read the bioluminescence signal without any additional step simplifies the procedures and improves both precision and reproducibility. Moreover, the system allows multiple measurements in the same sample, thus allowing kinetic studies where manipulations and errors are minimized. Another advantage of our bioluminescence-based assays is that the read out of each experiment is not dependent on the operator' skills, further strengthening the validity of this model. Although our study has been carried out only in vitro, future studies will focus on real-time monitoring of biofilm formation onto ETT pieces subcutaneously implanted in mice, similarly to what has been already done by Kucharikova and colleagues with CVC pieces [28].

In line with other bioluminescence analysis systems $[28,31,46]$, here we show that quantification of bioluminescence by RLU provides data in line with those obtained by conventional techniques for biofilm quantification. When compared to techniques such as CFU and $\mathrm{CV}$ determination, our method requires shorter processing times and it provides more accurate data for discriminating kinetic differences among different experimental groups. Moreover, morphological analysis of the ETT-associated biofilm has been evaluated by confocal microscopy. Finally, quantification of viable cells can be easily performed as well. Indeed, by this approach we have been able to easily discriminate between living and dead cells embedded in the ETT biofilm, therefore assessing the viability of biofilm-embedded bacteria, in the presence and in the absence of gentamicin. Also, the expression of $P$. aeruginosa virulence traits (like eDNA and pyoverdine production as well as quorum sensing genes expression levels) have been directly measurable in ETT-structured biofilm. To our knowledge, this in vitro model mimics as close as possible what may happen in an in vivo situation, such as in the ventilator-assisted patients.

\section{Conclusions}

Overall, we have described an original experimental model that, by providing a dynamic information on the development of a sessile microbial community onto medical devices, may find a rapid and practical application. Studies on the effectiveness of both new antimicrobial drugs as well as novel biomaterials, useful for designing and manufacturing innovative medical devices, may strongly benefit from such method. Therefore, the present model may find successful application not only in vitro but also in clinical studies.

\section{Additional files}

Additional file 1: Preparation of paediatric ETT pieces. A sterile disposable paediatric endotracheal tube (ETT) (RUSCH $3.0 \mathrm{~mm}-5.0 \mathrm{~mm}$ ) was cut in pieces of $0.5 \mathrm{~cm}$ length, using a biological safety cabinet to ensure sterile conditions. (TIF $4704 \mathrm{~kb}$ )

Additional file 2: Calibration curve of BLI-Pseudomonas bioluminescence detection. Increasing numbers of BLI-Pseudomonas cells grown in TSB with $2 \%$ sucrose at $37^{\circ} \mathrm{C}$ were analysed for bioluminescence emission by using luminometer. (PDF 80 kb)

Additional file 3: Evaluation of BLI-Pseudomonas biofilm on coverslips. The data represent the mean +/- SEM of BLI-Pseudomonas biofilm thickness (expressed in $\mu \mathrm{m}$ ) on five different fields of each coverslip, untreated (black columns) or treated (grey columns) with gentamicin, after 24,48 or $72 \mathrm{~h}$ of incubation. ${ }^{* * *} p<0.001 ; 24$ h-biofilm vs $72 \mathrm{~h}$ biofilm ${ }^{* * * *} p<0.0001$; gentamicin-treated biofilm vs untreated biofilm. (TIF 1096 kb)

Additional file 4: Analysis of biofilm-related genes. BLI-Pseudomonas quorum sensing genes (lasR/lasl, lasB, toxA, algD) in a 24 h-old biofilm formed on ETT pieces and in the planktonic cells are shown. Lanes show the bands of each gene, according to the length of fragments expected, detected by gel analysis: lasR: 725 bp; lasl: 605 bp; lasB 300 bp, toxA 352 bp and algD 1310 bp ( $L=$ ladder). Gentamicin-treated biofilm served as control. (PDF $50 \mathrm{~kb}$ )

\section{Abbreviations}

ANOVA: Analysis of variance; ATCC: American type culture collection; BLI: Bioluminescence; CFDA: 5(6)-carboxyfluorescein diacetate; CFU: Colony forming units; CV: Crystal violet; CVC: Central venous catheters; DIC: Differential interference contrast; eDNA: Extracellular DNA; EPS: Extracellular polymeric substance; ETT: Endotracheal tubes; FBS: Fetal bovine serum; OD: Optical density; PBS: Phosphate-buffered saline; PCR: Polymerase chain reaction; PFA: Paraformaldehyde; PI: Propidium iodide; QS: Quorum sensing; RFU: Relative fluorescence units; RLU: Relative luminescence units; RT: Room temperature; SEM: Standard error of the mean; TSA: Tryptic soy agar; TSB: Tryptic soy broth; VAP: Ventilator-associated pneumonia; WT: Wild type

\section{Acknowledgments}

The research has been done with the support of Nikon Instruments S.p.A., through the setup of the laboratory NIK@UNIMORE (Dr. Lucia Denti and Prof. Elena Bassoli).

\section{Funding}

EP was supported by FAR-2017.

\section{Availability of data and materials}

The datasets used and/or analysed during the current study are available from the corresponding author on reasonable request. 


\section{Authors' contributions}

EP primarily designed the study, conducted most of the analysis and wrote the manuscript. BC, GF, Rl, AS performed the laboratory work and conducted most of the analysis. AA, SP, EB helped to analyze the results and contributed to write the manuscript. MG provided the ETTs used for the study and contributed to write the manuscript. All authors read and approved the final manuscript.

\section{Ethics approval and consent to participate}

No applicable

\section{Consent for publication}

Not applicable

\section{Competing interests}

The authors declare that they have no competing interests.

\section{Publisher's Note}

Springer Nature remains neutral with regard to jurisdictional claims in published maps and institutional affiliations.

\section{Author details}

'Department of Surgical, Medical, Dental and Morphological Sciences with interest in Transplant, Oncological and Regenerative Medicine, University of Modena and Reggio Emilia, Modena, Italy. ${ }^{2}$ Department of Life Sciences, University of Modena and Reggio Emilia, Modena, Italy.

Received: 4 April 2018 Accepted: 30 July 2018

Published online: 14 August 2018

\section{References}

1. Larson E. Bacterial colonization of tracheal tubes of patients in a surgical intensive care unit. Nurs Res. 1970;19:122-8.

2. Inglis TJ, Millar MR, Jones JG, Robinson DA. Tracheal tube biofilm as a source of bacterial colonization of the lung. J Clin Microbiol. 1989;27:2014-8.

3. Feldman C, Kassel M, Cantrell J, Kaka S, Morar R, Goolam Mahomed A, et al. The presence and sequence of endotracheal tube colonization in patients undergoing mechanical ventilation. Eur Respir J. 1999;13:546-51.

4. Zur KB, Mandell DL, Gordon RE, Holzman I, Rothschild MA. Electron microscopic analysis of biofilm on endotracheal tubes removed from intubated neonates. Otolaryngol--Head Neck Surg Off J Am Acad Otolaryngol-Head Neck Surg. 2004;130:407-14.

5. Solomon DH, Wobb J, Buttaro BA, Truant A, Soliman AMS. Characterization of bacterial biofilms on tracheostomy tubes. Laryngoscope. 2009;1 19:1633-8

6. Silva RC, Ojano-Dirain CP, Antonelli PJ. Effectiveness of pediatric tracheostomy tube cleaning. Arch Otolaryngol Head Neck Surg. 2012;138:251-6.

7. Perkins J, Mouzakes J, Pereira R, Manning S. Bacterial biofilm presence in pediatric tracheotomy tubes. Arch Otolaryngol Head Neck Surg. 2004;130: 339-43.

8. Al-Samri M, Mitchell I, Drummond DS, Bjornson C. Tracheostomy in children: A population-based experience over 17 years. Pediatr Pulmonol. 2010:45:487-93.

9. Tarquinio K, Confreda K, Shurko J, LaPlante K. Activities of tobramycin and polymyxin E against Pseudomonas aeruginosa biofilm-coated medical grade endotracheal tubes. Antimicrob Agents Chemother. 2014;58:1723-9.

10. Gil-Perotin S, Ramirez P, Marti V, Sahuquillo JM, Gonzalez E, Calleja I, et al. Implications of endotracheal tube biofilm in ventilator-associated pneumonia response: A state of concept. Crit Care Lond Engl. 2012;16:R93.

11. Adair CG, Gorman SP, Feron BM, Byers LM, Jones DS, Goldsmith CE, et al. Implications of endotracheal tube biofilm for ventilator-associated pneumonia. Intensive Care Med. 1999:25:1072-6.

12. Strateva T, Mitov I. Contribution of an arsenal of virulence factors to pathogenesis of Pseudomonas aeruginosa infections. Ann Microbiol. 2011;61:717-32.

13. Flemming $\mathrm{H}-\mathrm{C}$, Wingender J. The biofilm matrix. Nat Rev Microbiol. 2010;8:623-33.

14. Ryder C, Byrd M, Wozniak DJ. Role of polysaccharides in Pseudomonas aeruginosa biofilm development. Curr Opin Microbiol. 2007;10:644-8.

15. Ghafoor A, Hay ID, Rehm BHA. Role of exopolysaccharides in Pseudomonas aeruginosa biofilm formation and architecture. Appl Environ Microbiol. 2011;77:5238-46.

16. Sutherland IW. The biofilm matrix--an immobilized but dynamic microbial environment. Trends Microbiol. 2001;9:222-7.
17. Allesen-Holm M, Barken KB, Yang L, Klausen M, Webb JS, Kjelleberg S, et al. A characterization of DNA release in Pseudomonas aeruginosa cultures and biofilms. Mol Microbiol. 2006;59:1114-28.

18. Whitchurch CB, Tolker-Nielsen T, Ragas PC, Mattick JS. Extracellular DNA required for bacterial biofilm formation. Science. 2002;295:1487.

19. Mulcahy H, Charron-Mazenod L, Lewenza S. Pseudomonas aeruginosa produces an extracellular deoxyribonuclease that is required for utilization of DNA as a nutrient source. Environ Microbiol. 2010;12:1621-9.

20. Hazan Z, Zumeris J, Jacob H, Raskin H, Kratysh G, Vishnia M, et al. Effective prevention of microbial biofilm formation on medical devices by low-energy surface acoustic waves. Antimicrob Agents Chemother. 2006;50:4144-52.

21. Kopel M, Degtyar E, Banin E. Surface acoustic waves increase the susceptibility of Pseudomonas aeruginosa biofilms to antibiotic treatment Biofouling. 2011;27:701-10.

22. Davies D. Understanding biofilm resistance to antibacterial agents. Nat Rev Drug Discov. 2003;2:114-22.

23. del Pozo JL, Patel R. The challenge of treating biofilm-associated bacterial infections. Clin Pharmacol Ther. 2007;82:204-9.

24. Garland JS. Strategies to prevent ventilator-associated pneumonia in neonates. Clin Perinatol. 2010;37:629-43.

25. Tablan OC, Anderson LJ, Besser R, Bridges C, Hajjeh R, CDC, et al. Guidelines for preventing health-care--associated pneumonia, 2003: recommendations of CDC and the Healthcare Infection Control Practices Advisory Committee. MMWR Recomm Rep Morb Mortal Wkly Rep Recomm Rep. 2004;53 RR-3:1-36.

26. Pan Y, Song S, Tang X, Ai Q, Zhu D, Liu Z, et al. Streptococcus sp. in neonatal endotracheal tube biofilms is associated with ventilator-associated pneumonia and enhanced biofilm formation of Pseudomonas aeruginosa PAO1. Sci Rep. 2017;7:3423.

27. Choi K-H, Schweizer HP. Mini-Tn7 insertion in bacteria with single attTn7 sites: Example Pseudomonas aeruginosa. Nat Protoc. 2006;1:153-61.

28. Kucharíková S, Vande Velde G, Himmelreich U, Van Dijck P. candida albicans biofilm development on medically-relevant foreign bodies in a mouse subcutaneous model followed by bioluminescence imaging. J Vis Exp JoVE. 2015;95:52239.

29. Stepanovic S, Vukovic D, Dakic I, Savic B, Svabic-Vlahovic M. A modified microtiter-plate test for quantification of staphylococcal biofilm formation. J Microbiol Methods. 2000:40:175-9.

30. Das MC, Sandhu P, Gupta P, Rudrapaul P, De UC, Tribedi P, et al. Attenuation of Pseudomonas aeruginosa biofilm formation by Vitexin: A combinatorial study with azithromycin and gentamicin. Sci Rep. 2016;6 https://doi.org/10.1038/srep23347.

31. Paulone S, Ardizzoni A, Tavanti A, Piccinelli S, Rizzato C, Lupetti A, et al. The synthetic killer peptide KP impairs Candida albicans biofilm in vitro. PLoS One. 2017;12:e0181278.

32. Dutka-Malen S, Leclercq R, Coutant V, Duval J, Courvalin P. Phenotypic and genotypic heterogeneity of glycopeptide resistance determinants in grampositive bacteria. Antimicrob Agents Chemother. 1990;34:1875-9.

33. Schaber JA, Carty NL, McDonald NA, Graham ED, Cheluvappa R, Griswold JA, et al. Analysis of quorum sensing-deficient clinical isolates of Pseudomonas aeruginosa. J Med Microbiol. 2004;53(Pt 9):841-53.

34. Lanotte P, Watt S, Mereghetti L, Dartiguelongue N, Rastegar-Lari A, Goudeau A, et al. Genetic features of Pseudomonas aeruginosa isolates from cystic fibrosis patients compared with those of isolates from other origins. J Med Microbiol. 2004:53(Pt 1):73-81.

35. Savli H, Karadenizli A, Kolayli F, Gundes S, Ozbek U, Vahaboglu H. Expression stability of six housekeeping genes: A proposal for resistance gene quantification studies of Pseudomonas aeruginosa by real-time quantitative RT-PCR. J Med Microbiol. 2003:52(Pt 5):403-8.

36. Sabia C, de Niederhäusern S, Guerrieri E, Messi P, Anacarso I, Manicardi G, et al. Detection of bacteriocin production and virulence traits in vancomycin-resistant enterococci of different sources. J Appl Microbiol. 2008;104:970-9.

37. Meyer JM, Neely A, Stintzi A, Georges C, Holder IA. Pyoverdin is essential for virulence of Pseudomonas aeruginosa. Infect Immun. 1996;64:518-23.

38. Banin E, Vasil ML, Greenberg EP. Iron and Pseudomonas aeruginosa biofilm formation. Proc Natl Acad Sci U S A. 2005;102:11076-81.

39. Bjarnsholt T, Ciofu O, Molin S, Givskov M, Høiby N. Applying insights from biofilm biology to drug development - can a new approach be developed? Nat Rev Drug Discov. 2013:12:791-808.

40. Lawrence JR, Korber DR, Hoyle BD, Costerton JW, Caldwell DE. Optical sectioning of microbial biofilms. J Bacteriol. 1991:173:6558-67. 
41. Malaty J, Antonelli PJ. Effect of blood and mucus on tympanostomy tube biofilm formation. Laryngoscope. 2008;118:867-70.

42. De Marco S, Piccioni M, Pagiotti R, Pietrella D. Antibiofilm and antioxidant activity of Propolis and bud poplar resins versus Pseudomonas aeruginosa. Evid Based Complement Alternat Med. 2017;2017:1-11.

43. Rodrigues ME, Lopes SP, Pereira CR, Azevedo NF, Lourenço A, Henriques M, et al. Polymicrobial ventilator-associated pneumonia: Fighting in vitro Candida albicans-Pseudomonas aeruginosa biofilms with antifungalantibacterial combination therapy. PLoS One. 2017;12:e0170433.

44. Enjalbert B, Rachini A, Vediyappan G, Pietrella D, Spaccapelo R, Vecchiarelli A, et al. A multifunctional, synthetic Gaussia princeps luciferase reporter for live imaging of Candida albicans infections. Infect Immun. 2009;77:4847-58.

45. Gahan CGM. The bacterial lux reporter system: Applications in bacterial localisation studies. Curr Gene Ther. 2012;12:12-9.

46. Vande Velde G, Kucharíková S, Schrevens S, Himmelreich U, Van Dijck P. Towards non-invasive monitoring of pathogen-host interactions during Candida albicans biofilm formation using in vivo bioluminescence. Cell Microbiol. 2014;16:115-30.

Ready to submit your research? Choose BMC and benefit from:

- fast, convenient online submission

- thorough peer review by experienced researchers in your field

- rapid publication on acceptance

- support for research data, including large and complex data types

- gold Open Access which fosters wider collaboration and increased citations

- maximum visibility for your research: over $100 \mathrm{M}$ website views per year

At $\mathrm{BMC}$, research is always in progress.

Learn more biomedcentral.com/submissions 